\title{
Developmental Effects of Malathion Exposure on Recognition Memory and Spatial Learning in Males Wistar Rats
}

\author{
Pacôme Kouadio N'Go ${ }^{1 *}$, Fatima-Zahra Azzaoui ${ }^{1}$, Porlo Rigobert Soro ${ }^{1}$, Majda Samih ${ }^{1}$, \\ Ahmed Omar Touhami Ahami ${ }^{1}$, Mohamed Najimi ${ }^{2}$, Fatiha Chigr ${ }^{2}$ \\ ${ }^{1}$ Unit of Clinics and Cognitives Neurosciences, Laboratory of Biology and Health, Department of Biology, \\ Faculty of Sciences, University Ibn Tofail, Kénitra, Morocco \\ ${ }^{2}$ Laboratory of Physiology Animal, Department of Biology, Faculty of Sciences and Technology, \\ University Sultan Moulay Slimane, Béni Mellal, Morocco

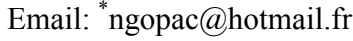

Received March 25, 2013; revised April 26, 2013; accepted May 9, 2013

Copyright (C) 2013 Pacôme Kouadio N'Go et al. This is an open access article distributed under the Creative Commons Attribution License, which permits unrestricted use, distribution, and reproduction in any medium, provided the original work is properly cited.

\begin{abstract}
Most cognitive effects of Organophosphate Pesticides (OP) are induced after exposure to parathion, chlorpyrifos and diazinon, which the usage has been restricted because of overt signs of their toxicities. In this study, we investigate whether developmental exposure to Malathion could impair spatial learning and recognition memory in male rats. Animals exposed by intragastric route, from in utero to young adult stage, to incremental doses of Malathion dissolved in corn oil; 100, 200 and $300 \mathrm{mg} / \mathrm{kg}$ of body weight, and one control group are given corn oil. Then, cognitive and behaveioral abilities are assessed using Barnes maze and object recognition memory task. Malathion administration at 300 $\mathrm{mg} / \mathrm{kg}$ is toxic to pregnant dams, and pups are stillborns. Rats exposed to $200 \mathrm{mg} / \mathrm{kg}$ make a significant working memory error, and require more time to find an escape box during the initial training phase of Barnes maze. However, fewer errors are made in rats exposed to $100 \mathrm{mg} / \mathrm{kg}$. For reversal learning task, the high dose group shows great deficits in spatial strategy to locate the new position of the box. With respect to recognition task, both dose $100 \mathrm{and} 200 \mathrm{mg} / \mathrm{kg}$ impair significant short-term ( $2 \mathrm{~h}$ after habituation phase) object recognition memory, but long-term $(24 \mathrm{~h}$ after habituation phase) recognition memory is intact in high dose group. The current study also reveals that all treatments induce high significant neocortex acetylcholinesterase (AChE) activity inhibition, but $100 \mathrm{mg} / \mathrm{kg}$ dose is not sufficient to disrupt great hippocampal activity alteration. These results suggest that developmental exposure to Malathion, despite low toxicity described, may induce late-emerging spatial learning and recognition memorialterations. Moreover, Cortical and hippocampal area that support strongly these behaviors remain sensitive to incremental doses of Malathion.
\end{abstract}

Keywords: AChE; Developmental Neurotoxicity; Malathion; Organophosphate Pesticide Recognition Memory; Spatial Learning

\section{Introduction}

Organophosphate insecticides (OP) are widely used in agriculture pest control in order to improve the quantity and quality of food production. However, they are also well-known as environmental contaminants occurred in crop products, water supplies, and in air. Thus, OPs exposure remains a factor of human hazard health, especially in young children population [1-3]. The Malathion insecticide is one of the most used OP in United States and throughout the world. As evoked by Maroni et al.,

${ }^{*}$ Corresponding author.
Malathion is used in agriculture, veterinary practice and as ectoparasiticide applied against human body lice [4]. From chemical name [S-1,2(bis-ethoxycarbonyl)ethylO, O-dimethyl phosphorodithioate], Malathion exerts, through bioactive form malaoxon, its primary effect by phosphorylating the serine residue at the active site of acetylcholinesterase (AChE) [5]. This leads to an inhibition of this enzyme in neural tissues, and so resulting overstimulation of cholinergic synapse in central and peripheral nervous system [6,7]. Although Malathion is considered as an OP relatively low acute toxicity, it could cause others high toxic OPs, a great risk for human 
and environmental health. Several studies have examined the possible association between occupational exposure to pesticides and certain types of cancer, particularly non-Hodgkin's lymphoma (NHL) and leukemia. It is difficult to establish association with specific pesticides because workers are exposed chronically to multiples compounds. However, exposure to Malathion was associated with increased risk of NHL in a study of men in Iowa and Minnesota [8], of women in Nebraska [9], and of men in Canada [10]. Moreover, in California, Malathion was the third most frequently reported pesticide and caused five times more occupational illness than the average pesticide [11]. Many biochemical and physiological effects of OP insecticide have been documented in man and animal. Malathion exposure has been shown to significantly decrease the sperm count of mice [12]. Aother study revealed that Malathion in subchronic exposure affects rat hepatic gluconeogenesis and glycogenolysis as well as inducing hyperglycemia [13]. Furthermore, the main target of OPs insecticide is the nervous system, and it has been suggested that chronic exposure to low-level of OP can interfere with normal neurodevelopment [14]. Clinical studies support the observation that cognitive, neurobehavioral deficits, and neurology diseases occurre in agricultural communities using widely OPs insecticides $[15,16]$. In rats, previous study has shown that repeatedly exposure to Malathion induced depression-like behavior in Forced Swimming Test [17]. Acker, et al. [18] have also reported a decrease of motor coordination and muscular strength in rat pups exposed orally to Malathion, from post natal days (PND) 12 to 14 . However, the most toxicology studies are focused on high toxic OP compounds such as Chlorpyrifos (CPF), Diazinon (DZN) and Methyl parathion (MPS). Their common effects on cholinergic system and its biomarker molecules have been demonstrated [19-21]. In addition, behavioral studies revealed that early postnatal exposure to $\mathrm{CPF}$ and MPS impaired memory functions [22,23]. In contrast, scarce studies in this sense have been done for Malathion compound.

The interest of the current study is to assess recognition memory and spatial learning abilities in young male rats developmentally exposed to Malathion (i.e. from in utero through adolescence stage). We investigate also on AChE activity inhibition from hippocampal and neocortex area. Because, no studies have reported neurocognitive effects after developmental exposure to Malathion, discussion will made relative to high toxic OPs mentioned.

\section{Materials and Methods}

\subsection{Chemical}

Malathion was obtained from commercial grade: Maly- phos 50 (active ingredients $500 \mathrm{~g}$ by liter). The Malathion concentration ( $50 \%$ purity) in commercial grade was diluted in corn oil. Acetylthiocholine iodide, 5,5-dithiobis-(2-nitrobenzoic acid) (DTNB) and others all chemicals were purchased from Sigma (USA).

\subsection{Animals and Treatment}

Twelve Wistar female rats, 4 months of age were obtained from a local breeding colony of Faculty of Sciences, Kenitra-Morocco. Rates were kept under standard condition, $12 \mathrm{~h} \mathrm{light} / 12$ dark cycle, $20^{\circ} \mathrm{C} \pm 2{ }^{\circ} \mathrm{C}$ and $50 \%$ - 70\% humidity). They had access to commercial diet (ALF SAHEL-Casablanca, Morocco) and tap water ad libitum. After 2 weeks of acclimation, virgin rates and one-non-pesticide exposed male were bred in propylene cage covered by stainless steel mesh $(70 \times 40 \times 65 \mathrm{~cm})$. On gestational days six (GD 6), females were randomly divided in four groups of treatment. Then, three groups received by intragastric gavage incremental doses of Malathion insecticide dissolved in corn oil; 100, 200 and $300 \mathrm{mg} / \mathrm{kg}$ of body weight per day (Mal 100, Mal 200, Mal 300), and one control group (VEH) was given corn oil daily. Gestating females were treated from GD6 though post-partum day 21, rat pups were so exposed to Malathion via their mothers. On PND 21, weaned offsprings of each experimental group were submitted to similar dose regimens of preliminary protocol, and duration of treatment was extended to young adult stage (PND 45 - 60). Experimental procedures are also examined and approved by the internal ethical committee for animal welfare.

\subsection{Physical Parameter Measurements}

Beginning on PND1, physical signs of toxicity and body weight were daily recorded during treatment.

\subsection{Behavioral Assessment}

On PND60, after Malathion treatment period, recognition memory, spatial and learning memory were tested using the Novel object recognition task and Barnes Maze.

\subsubsection{Novel Object Recognition (NOR) Task}

The apparatus and procedure has been described elsewhere $[24,25]$. The maze took place in open field box of polywood $\left(50 \times 50 \times 40 \mathrm{~cm}^{3}\right)$, illuminated with halogen lamp $(60 \mathrm{~W})$. The NOR task is based on spontaneous tendency of animal to more interact with the novel object than the familiar. All rats were submitted to a habituation session in which they have freely explored the empty open field arena for $5 \mathrm{~min}$. On following day, each rat is initiated to familiarization phase during $5 \mathrm{~min}$. It consist to an exploration of two identical objects $(\mathrm{A}+\mathrm{A})$ positioned in two adjacent corners from $10 \mathrm{~cm}$ from the 
walls. In short-term memory (STM) test $2 \mathrm{~h}$ after familiarization phase, the rat is allowed $5 \mathrm{~min}$ to explore the field with a familiar object (A) and novel object (B) at the same position. Long-term memory (LTM) study is performed $24 \mathrm{~h}$ after familiarization phase; rat is led to visit again the apparatus in presence of the same familiar object (A) and other novel (C). Single set of three objects $(\mathrm{A}, \mathrm{B}, \mathrm{C})$ presented similar texture but distinctive in color, sharps and size. The arena and objects are cleaned with ethanol $70 \%$, after each training session to remove the olfactory cues. The Recognition Index (RI) indicates the ratio of novel object exploration time to the total of both novel and familiar object exploration time. It is the main index of retention. The concept of exploration of an object is defined as directing the nose at distance $\leq 1 \mathrm{~cm}$ to the object, and/or touching it with the nose. In contrast, turning around, sitting and climbing on the object was not considered as exploratory behavior.

\subsubsection{Barnes Circular Maze}

Memory and spatial learning were assessed in rodents with Barnes circular maze [26]. The apparatus and procedure adapted was a modification of Fedorova, et al. [27] and Greferath, et al. [28]. The maze consists of a white circular platform, $122 \mathrm{~cm}$ in diameter, raised to $50 \mathrm{~cm}$ above the floor level. The platform contains 18 holes (of $9 \mathrm{~cm}$ in diameter) evenly spaced around the circumference, but one of the holes was only connected to a removable escape box. Three visual cues on the walls, 50 $\mathrm{cm}$ around the maze, act as spatial cues. Each rat was preliminary given to one habituation session with the maze and existence of escape box, which the position remained constant throughout testing for a given animal. Habituation began by placing the rat in the center of the maze under a bucket in a room that was brightly lit (with halogen lamps of $500 \mathrm{~W}$ installed in the ceiling). After $10 \mathrm{~s}$, the bucket was lifted and the rat was guided to locate escape box, then the buzzer was turned off and rat was allowed to remain for $1 \mathrm{~min}$. On following days the habituation phase, initial training were conducted during 4 consecutive days ( 2 trial per days), under the same condition above, except that the rats needed to locate the escape box by themselves. Each trial ended when the rat entered the goal tunnel or after $5 \mathrm{~min}$ elapsed. There was a $3 \mathrm{~min}$ inter-trial interval for each animal. Three weeks after training phase, the rats were subjected to locate a new position of escape hole opposite to original one during 5 consecutive days; that for testing the reversal learning ability. The maze was routinely cleaned with ethanol solution $20 \%$ after every trial to avoid olfactory cues. Trials were recorded with video track Logitech. Training was conducted using a working memory paradigm, that was evaluated by the total number of errors made and the escape latencies time associated [29,30]. With respect to the reversal learning, we took also account the number of perseveration to explain deficits of news strategies elaboration. Perseverations were defined as repeat visits to the original hole or one of the two adjacent holes.

\subsection{AChE Activity Assay}

Rats are killed by decapitation $24 \mathrm{~h}$ after behavioral tests. Samples of brain correspondent to neocortex and hippocampus areas are removed and homogenized in buffer Tris/HCl (50 mmol/L, pH 7.3) and Sucrose (0.32 mol/L). The homogenate is centrifuged at $1000 \mathrm{xg}$ for $15 \mathrm{~min}$ at $4^{\circ} \mathrm{C}$. AChE activity is assayed according to Elman method [31], using acetylthiocholine iodide as a substrate. Reaction mixture contained $100 \mu \mathrm{L}$ of supernatant, $4 \mu \mathrm{L}$ of substrate $(75 \mathrm{mM}), 15 \mu \mathrm{L}$ of Dithiobisnitrobenzoic acid (DTNB as Ellman's reagent, $100 \mathrm{mM}$ ) and $3.0 \mathrm{ml}$ of phosphate buffer ( $\mathrm{pH}$ 8.0). The rate of Acetylthiocholine iodide hydrolysis is measured at $412 \mathrm{~nm}$ in spectrophotometer for $10 \mathrm{~min}$. Brain AChE is expresses in percent of inhibition from control group [32].

\subsection{Statistical Analysis}

All data were expressed as means \pm S.E.M (Standard Error of Mean). Repeat measure and one-way analyses of variance (ANOVA) are used to assess difference between groups in physical and neurobehavior performances induced by developmental exposure to Malathion. Post hoc comparisons were made using Tukey's HDS test, when appropriate. Statistical significant was assumed at $p<$ 0.05 .

\section{Results}

\subsection{General Physical Observations}

Malathion exposure at dosage $300 \mathrm{mg} / \mathrm{kg}$ (b.w) induced toxicity to pregnant dams, alls offsprings are stillborns during parturition. For others treated groups, no sign of toxicity such as body tremor, salivation, weakness and convulsion are observed. Repeated measure ANOVA shows a significant change in body weight gain between male rats study groups $(p=0.02)$. Tukey's post hoc test reveals that body weight in both treated-groups, "Mal 100 " and "Mal 200", is significantly reduced $(p<0.001)$ compared to $\mathrm{VEH}$, but no difference is observed between them (data not shown).

\subsection{Object Recognition Memory}

In STM phase, the mean of RI (Recognition Index) is above the threshold of object recognition $(50 \%)$ in VEH group. However, both treated-groups, Mal 100 and Mal 200 display similar performance on object exploration time; their RI mean is widely under the threshold of recognition (41\%). One-ways ANOVA shows that the dif- 
ference observed is statistically significant $[\mathrm{F}(2,19)=$ 200, 38; $p<0.001]$. During LTM recognition testing, rats of Mal 100 group have enhanced their RI compared to that performed in STM phase. In contrast, the RI remains sensibly unchanged in rats of Mal 200 group, from STM to LTM session (Figure1).

\subsection{Memory and Spatial Learning}

Developmental exposure to Malathion impairs significantly spatial memory in Barnes maze. During initial training period, rats of Mal 200 group perform better the first days than the others, as indicated by a reduction in latency time to find the escape box (Figure 2(a)). On 3 following days, the control rats learn the escape box position faster than treated-groups. Repeated measures ANOVA shows the latencies time has increased significantly for Mal 100 and Mal 200 treated-rats $[\mathrm{F}(2,19)=$ $50.7 ; p<0.001$ ], compared to VEH. However, no significant difference is observed between both treated groups $(p>0.05)$ (Figure 2(a)). The increase of latencies times in treated-groups is accompanied by significant working memory errors committed. In initial training phase, the number of errors increases during for 4 testing days, in Mal 200 group. But, errors made by Mal 100 group are remained sensibly constant during this period. Repeated measures ANOVA revealed a high significant effects of Malathion treatment on working memory $[\mathrm{F}(2,19)=197$. $80 ; p<0.001]$. Tukey's post hoc test confirms that these effects are significant in Malathion treated-groups $(p<$ 0.001 ), compared to control group. It also indicates that working memory impairment is more pronounced in Mal 200 group (Figure 2(b)).

With respect to reversal learning phase, the Malathion treated-rats express again more difficulties to learn a new position of the escape box, opposite to original one. Rats of each study group began the firth trial day by taking similar time to find rapidly the escape box. But, we observe high significant increase of latencies time during the second, third and fourth days in treated-groups $[\mathrm{F}(2$, $19)=71,58 ; p<0.001]$. Tukey's post hoc test detects that control group are required lesser time to find the box than treated-groups $(p<0.001)$. However, rats of "Mal 100 " group have enhanced significantly their learning abilities compared to those of "Mal 200" $(p<0.01)$ (Figure 3(a)). As working memory errors made in initial training phase, more errors are occurred in treated-groups compared to control group. There are an increasing trend of number of errors committed in reversal learning phase with the regimen dose $[\mathrm{F}(2,19)=39,00 ; p<0.001]$. Although, both treated-groups displayed similar number of errors on trial day one, the number of errors was significantly different in mal 100 group and compared to that of Mal 200, on following days $(p<0.001)$ (Figure 3(b)). Moreover, latency time and rate of errors number
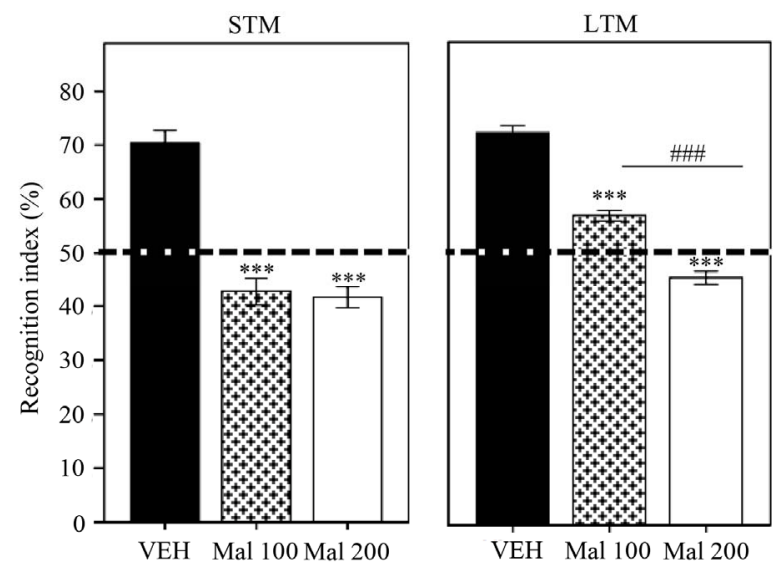

Figure 1. Effect of developmental neurotoxicity to Malathion on object recognition memory (Mal100 and Mal200 represent 100 and $200 \mathrm{mg} / \mathrm{kg}$; p.o, respectively. Data express percent of object recognition index as means \pm S.E.M of 7 - 8 animals per group. STM, Short Term Memory recognition ( $2 \mathrm{~h}$ after habituation phase); LTM, Long Term Memory recognition (24 $\mathrm{h}$ after habituation phase). ${ }^{* * *} \mathrm{p}<$ 0.001, comparison between exposed groups and VEH; ${ }^{\# \# p<}$ 0.001 as comparison between Mal 100 and Mal 200 groups (One-way ANOVA/Tukey's post-hoc analysis).

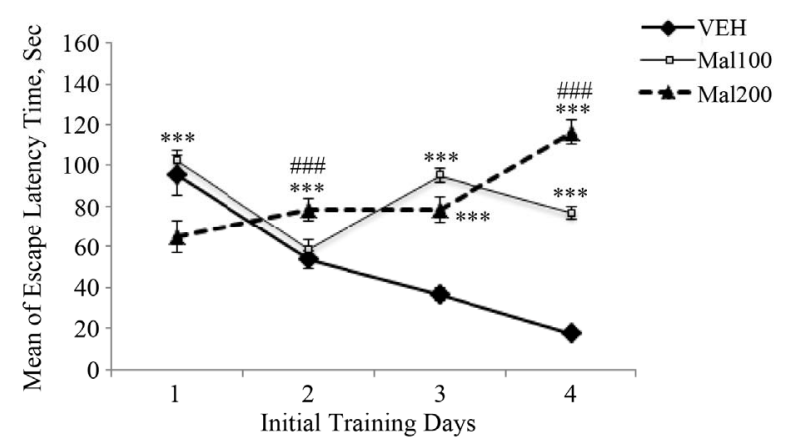

(a)

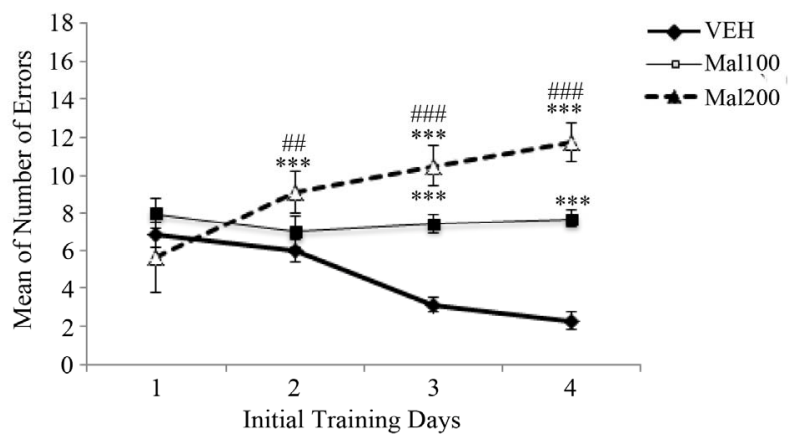

(b)

Figure 2. Initial training phase in Barnes maze. (a) Escape latencies times. (b) Number of Errors committed. Value are expressed in Means \pm S.E.M of 7 - 8 animals per group activities (Mal100 and Mal200 represent 100 and $200 \mathrm{mg} / \mathrm{kg}$; p.o, respectively). Developmental exposure to Malathion induced significant effects on initial spatial learning. ${ }^{* * * *} p<$ 0.001 as comparison to VEH. ${ }^{\#} p<0.01,{ }^{\# \# p}<0.001$ as comparison between Mal 100 and Mal 200 groups (Repeated measures ANOVA/Tukey's post-hoc analysis). 
elevated in reversal learning have associated to high number of perseveration, in treated-group (Figure 3(c)). Repeated measure ANOVA shows a significant difference in number of perseveration between all groups $[\mathrm{F}(2$, $19)=10,85 ; p<0.001)]$. Tukey's post hoc testdetectsthat the perseveration behavior increased significantly in "Mal 100" and "Mal 200" groups $(p<0.001)$

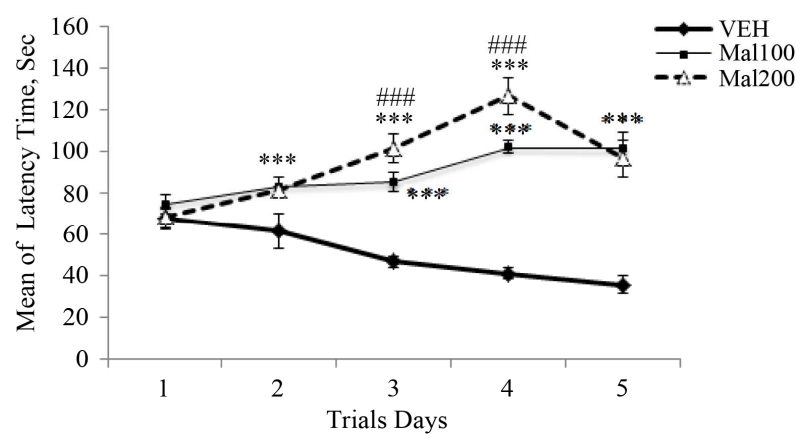

(a)

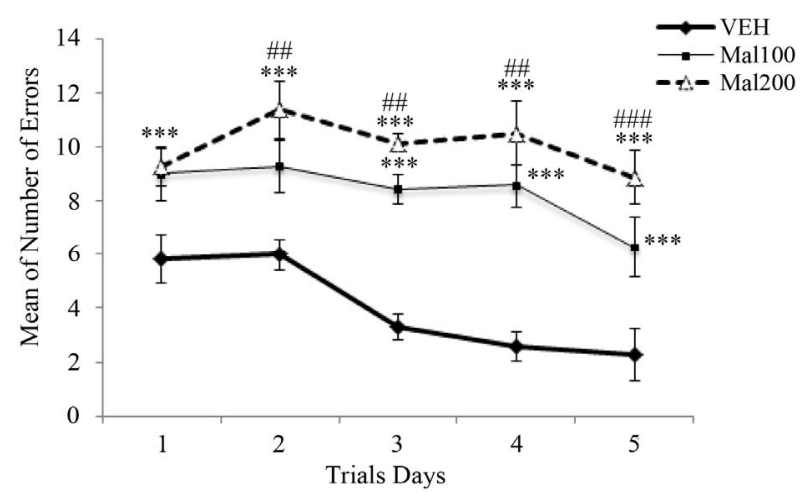

(b)

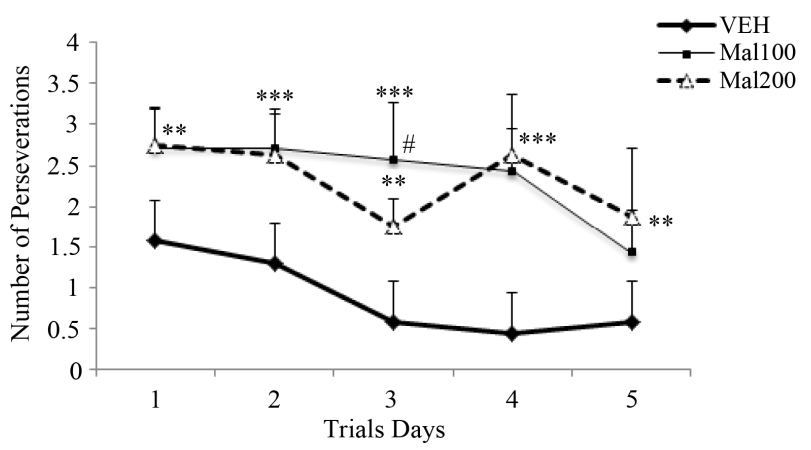

(c)

Figure 3. Reversal learning phase in Barnes maze. (a) Escape latencies times Latency time. (b) Number of Errors committed. (c) Number of Perseverations. Values are expressed as Means \pm S.E.M of 7 - 8 animals per group activities (Mal100 and Mal200 represent 100, $200 \mathrm{mg} / \mathrm{kg}$; p.o, respectively). Developmental exposure to Malathion induced significant effects on reversal spatial learning. ${ }^{* *} p<$ $0.01,{ }^{* * * *} p<0.001$ as compared to VEH; ${ }^{\#}<0.05,{ }^{\#} p<0.01$; $\#$ \#\#\# $<.001$ as comparison between Mal100 and Mal200 groups (Repeated measures ANOVA/Tukey's post-hoc analysis). compared to VEH, but no difference between them (Figure 3(c)).

\subsection{AChE Activity Assays}

Developmental exposure of Malathion to doses 100 and $200 \mathrm{mg} / \mathrm{kg}$ increases significantly neocortex AChE inhibition activity; $+26 \%(p<0.001)$ and $+46 \%(p<0.001)$ respectively, relative to control. Turkey post-hoc analysis detected that there is also great statistical difference between Mal 100 and Mal 200 groups $(p<0.01)$. We also found a significant AChE activity inhibition in hippocampus $+36 \%$ for "Mal 200" group $(p<0.001)$ and +20 for Mal 100 group $(p<0.01)$, compared to the control group. However, a significant change of AChE activity is observed in Mal 200, relative to Mal 100 ( $p<0.01)$, (Figure 4).

\section{Discussion}

In current study, except lethal dose of $300 \mathrm{mg} / \mathrm{kg}$, our result indicates that developmental exposure to Malathion does not induce general signs of systemic toxicity as weakness, tremor or convulsion. However, doses of 100 and $200 \mathrm{mg} / \mathrm{kg}$ affected body weight in treated-rats significantly compared to control. Current outcome is consistent with that of past study showing significant reduction of body weight in young rats exposed to parathion (potential OP toxic) at PND8-20 [33]. This could probably due to the effect of Malathion as others OPs, which are caused cholinergic over stimulation, followed
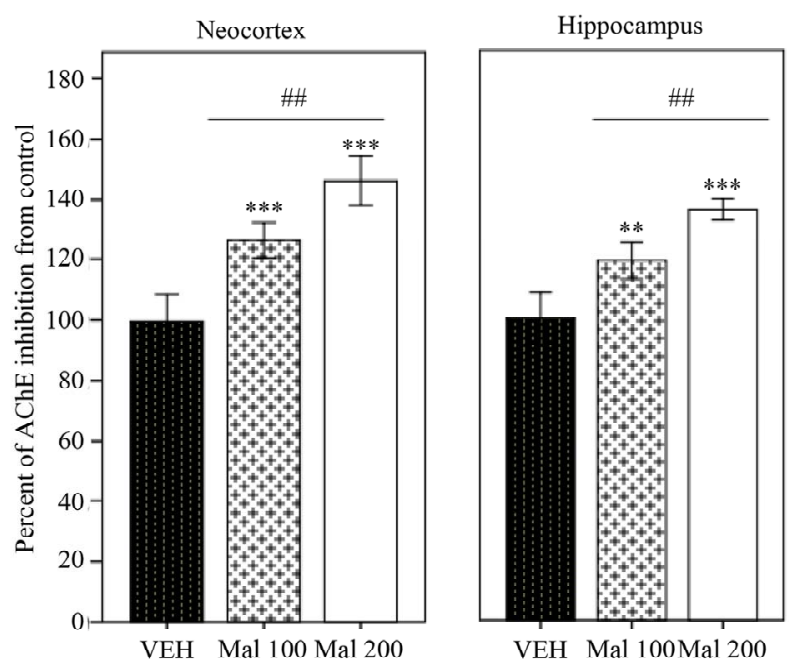

Figure 4. Effect of Malathion exposure on cerebral cortex and Hippocampus AChE activity inhibition (Mal100 and Mal200 group receive 100 and $200 \mathrm{mg} / \mathrm{kg}$; p.o, respectively). Data expressed percent of AChE change from VEH (Mean \pm S.E.M of $7-8$ animals per sex's group). ${ }^{* * *} p<0.01$ and ${ }^{* * * *} p<$ 0.001 as compared to VEH; ${ }^{\# \#}<<0.01$ as comparison between Mal 100 and Mal 200 groups (One-Way ANOVA/ Tukey's post-hoc analysis). 
by an increase of gastric motility and reduction of digestive tract absorption [34]. In contrast, recent study has revealed no weight loss in rats developmentally exposed (PND11-14) to the same dose of Malathion to ours [18]. These findings suggest that prenatal phase could be a sensitive period to developmental effects of Malathion on physical appearance.

Furthermore, the current experiment has shown that developmental exposure of Malathion disrupts recognition memory forming. In STM recognition task, both treated groups have spent lesser times to explore novel object than the control group. However, rats of Mal 200 group are significantly affected during long-term object recognition memory phase, relative to those of Mal 100 group. The under performances in rats receiving 200 $\mathrm{mg} / \mathrm{kg}$ of Malathion are associated with high hippocampal AChE activity inhibition, evaluated at young adult stage. That reinforces the hypothesis that cerebral AChE inhibition caused by Malathion in rat pups may induce cognitive impairments, later in life. In fact, the object recognition test is based on the discrimination between familiar and novel stimulus and natural performance of subject who need to respond to "what" stimulus was used in experiment previously. Hippocampus plays so a pivotal role in encoding and consolidation of novel stimulus; this process leads to integration and reorganization of the already formed memories. Thereby, when an object is previously encountered and reactivated later, the hippocampus allows the discrimination between the old and novel object, and followed to the natural tendency of novelty preference [35]. In addition, the recognition index is significantly reduced during long-term phase evaluation, in group treated with $200 \mathrm{mg} / \mathrm{kg}$. This index reflects the main index of object's recollection in the sense of familiarity with the feature of that particular stimulus. According to Reger, et al. [36], hippocampus is involved in long-term object recognition. Thus, the long-lasting object recognition disability could be due to the significant alteration of hippocampus AChE activity. Our findings are consistent with a recent study that revealed the impairment of hippocampus-dependent object recognition in neonatal rats developmentally exposed to diazinon OP [37]. Besides, short-term object recognition has been linked to other medial temporal area as the perirhinal cortex [38]. Our results support that both level of 100 and $200 \mathrm{mg} / \mathrm{kg}$ induce significant deficits of object recognition memory, in rats evaluated $2 \mathrm{~h}$ after training phase. That leads to suggest that perirhinal cortex was inherently more vulnerable to developmental exposure of Malathion. In fact, perirhinal cortex is the first forebrain site of several environment information's entrance as visual, olfactory, and somatosensory stimulus, in which hippocampus receives inputs [39]. The important cholinergic transmission within perirhinal cortex also seems to play a critical role in object recognition [40]. During brain development, acetylcholine and cholinergic projections play a major role in proliferation, migration, synaptogenesis and in normal development of neural cell cytoarchitecture $[41,42]$. However, it has been demonstrated that developmental exposure of OP including Malathion can interfere with the neurotransmitter function leading to neurodevelopment abnormalities $[43,44]$. Considering that Malathion induced short-term object recognition deficit thought cholinergic system alteration, one possible explanation is resided in Slotkin, et al. study. They have pointed that neonatal rats exposed to parathion on PND 1-4 showed widespread abnormalities in cholinergic synaptic markers encompassing effect on brain region possessing cholinergic projection and cell bodies [19]. Although Malathion is relatively less hazardous toxicant (orally LD50 800 - $1000 \mathrm{mg} / \mathrm{kg}$ ), neurocognitive deficits could explain to long-term exposure of rat pups in current study. Eventual persistence effects of Malathion on cholinergic functioning can so recovery those of high toxic OPs. Moreover, past study has reported evidences of a possible involvement of hippocampal serotonin (5-HT) neurotransmission in memory processing, with using NOR test [45]. It has been shown that CPF insecticide OPs target serotonin synaptic neurochemistry $[46,47]$. So, it could not exclude other noncholinergic effects on the neurobehavioral performances.

We have also investigated the effects of developmental exposure to Malathion on learning and spatial memory performances. The choice of Barnes maze has been done because it is less stressful and physically less taxing than others spatial mazes [48]. Our results show that initial spatial acquisition performances are significantly reduced during training days in rats exposed to both 100 and 200 $\mathrm{mg} / \mathrm{kg}$ (Figure 2(a)). They have expressed more time to find the escape box. That is accompanied by a significant increase of working error's number in rats of Mal 200 group. In fact, the initial acquisition as well as /or spatial memory consolidation seem heavily hippocampus-dependent, yet animal well-performance on Barnes maze is based on hippocampus functional integrity $[49,50]$. Similar to our findings, previous study reported significant spatial acquisition disruption in male rats exposed to CPF, from in utero through early in life [22]. These deficits could be due to significant hippocampal AChE activity inhibition observed in cholinergic synapse of rats treated with Malathion. The catalytic function of AChE in neuronal transmission takes place when cholinergic synapses are clearly established. However, other role in neuronal morphogenic has been demonstrated [51]. During brain development, AChE plays an adhesion molecules role, and interacts with neurexin proteins leading to neural cytoarchitecture changes [52,53]. In addition, these behavioral 
underperformances could be explained by Qiao, et al. study which reported evidences of synaptic cholinergic defects and more hippocampal cell biomarkers alteration, in neonatal rats exposed to CPF [54]. It has been also suggested that OP including Malathion exposure may change neuronal connectivity in the developing brain [52]. Previous study showed that Malathion exposure induces decrement effect on dendritic morphology of hippocampal CA1 neurons [55]. Thus, the reduction of neurogenesis rate could be a major explanation of disruption of spatial memory consolidation, as evoked by Sarkisyan, et al [56]. We find also that Malathion level at 200 $\mathrm{mg} / \mathrm{kg}$ appeared to produce greater deficits in working memory than did $100 \mathrm{mg} / \mathrm{kg}$. Our outcomes are consistent with previous researches that reported only high exposure OP including CPF induced impairment of working memory in male rats $[22,57]$. In animals exposed to low level of Malathion, the hippocampal AChE inhibition is slower than the rats exposed to high dose. However, they are performed significantly worse than control. That may suggest that other non-cholinergic mechanisms are involved in initial spatial learning.

After 3 weeks from initial training days, we have assessed rat's ability to learn a new location of the escape, in reversal learning task. This task requires learning new search strategies by suppressing the execution of the previous learning. It also evaluates also adaptive behaviors of rodents in novel environmental situation. In current study, rats developmentally exposed to both levels of Malathion have expressed great difficulties to learn new strategies (Figures 3(a) and 3(b)). That is associated to a number of errors and perseveration significantly elevated, relative to controls. However, the rats treated with low level of Malathion have performed better. In reversal learning task, spatial strategies do not require only involve menthippocampus functional integrity, but also prefrontal cortex and nucleus accumbens [58,59]. Prefrontal area is an important component of frontal lobe involved in conception, choice and execution of a planning. In Barnes maze, the selection of news spatial strategies are based on spatial memory that requires control executive processes like attention. But, the accumbens nucleus has been identified as a critical site in the neuronal circuit controlling motivation and mood [60], and mediating adaptive behaviors in rats [61]. In current study, we have found that developmental exposure to Malathion elicited significant cholinergic activity changes in cerebral cortex area. Aother study finds similar finding in frontal cortex in males rats exposed to DZN [62]. In additional, Campaña, et al. [55] reported evidences of significant decrement on dendritic morphology in prefrontal cortex neurons (PFC), also on the dendritic spine density from nucleus accumbens, in mice exposed to Malathion. The inactivation of PFC lead to impairment of reversal learning in the rats when they ceased the using of previously acquired responses, as described in visual discrimination reversal learning task [63]. Besides, other authors have reported the reduction in dopaminergic transmission system in ventral striatum produced reversal learning deficits. In fact, accumbens nucleus is a component of striatum area that is implicated in reward, motivation (ventral part), motor skills and cognitive control (dorsal part), specifically in learning of stimulusresponse association [64,65]. Repeat exposure to paraquat OP is well known to trigger neurodegeneration of dopaminergic system in nigra substantial, the principal provider of dopamine in striatum [66].

From these current findings, developmental exposure to Malathion leads to spatial learning and object recognition memory impairments, in male rats' studies. The behavioral deficits could be explained by brain AChE activity disruption induced in treatment groups. This animal model's study may reinforce the hypothesis of relationship between environmental toxicant and neurodegenerative disease occurrence in agricultural communities. However, some immunohistochemistry investigations are required to elucidate further results obtained.

\section{Acknowledgements}

This study was supported by GDRI Neuro and N£uromed consortium.

\section{REFERENCES}

[1] B. Eskenazi, A. R. Marks, A. Bradman, K. Harley, D. B. Barr, C. Johnson, et al., "Organophosphate Pesticide Exposure and Neurodevelopment in Young Mexican-American Children," Environmental Health Perspectives, Vol. 115, No. 5, 2007, pp. 792-798.

[2] C. Lu, K. Toepel, R. Irish, R. A. Fenske, D. B. Barr and R. Bravo, "Organic Diets Significantly Lower Children's Dietary Exposure to Organophosphorus Pesticides," Environmental Health Perspectives, Vol. 114, No. 2, 2006, pp. 260-263.

[3] M. K. Morgan, L. S. Sheldon, C. W. Croghan, P. A. Jones, G. L. Robertson, J. C. Chuang, N. K. Wilson and C. W. Lyu, "Exposures of Preschool Children to Chlorpyrifos and Its Degradation Product 3,5,6-trichloro-2-pyridinol in Their Everyday Environments," Journal of Exposure Analysis and Environmental Epidemiology, Vol. 15, No. 4, 2005, pp. 297-309.

[4] M. Maroni, C. Colosio, A. Ferioli and A. Fait, "Biological Monitoring of Pesticide Exposure: A Review. Introduction," Toxicology, Vol. 143, No. 1, 2000, pp. 1-118.

[5] D. J. Ecobichon, "Pesticides and Neurological Diseases," CRC Press, Boca Raton, 1994, p. 381.

[6] L. M. Wang, W. H. Ye, S. S. Zhou, K. D. Lin, M. R. Zhao and W. P. Liu, "Acute and Chronic Toxicity of Organophosphate Monocrotophos to Daphnia Magna," Journal of Environmental Science and Health, Part B, 
Vol. 44, No. 1, 2009, pp. 38-43.

[7] T. C. Kwong, "Organophosphate Pesticides: Biochemistry and Clinical Toxicology," Therapeutic Drug Monitoring, Vol. 24, No. 1, 2002, pp. 144-149. doi:10.1097/00007691-200202000-00022

[8] K. P. Cantor, A. Blair, G. Everett, et al., "Pesticides and Other Agricultural Risk Factors for Non-Hodgkin's Lymphoma among Men in Iowa and Minnesota," Cancer Research, Vol. 52, No. 9, 1992, pp. 2447-2455.

[9] S. H. Zahm, D. D. Weisenburger, R. C. Saal, et al., "The Role of Agricultural Pesticide Use in the Development of Non-Hodgkin's Lymphoma in Women," Archives of Environmental Health, Vol. 48, No. 5, 1993, pp. 353-358. doi:10.1080/00039896.1993.9936725

[10] H. H. McDuffie, P. Pahwa, J. R. McLaughlin, et al., "Non-Hodgkin's Lymphoma and Specific Pesticide Exposures in Men: Cross-Canada Study of Pesticides and Health," Cancer Epidemiology, Biomarkers \& Prevention, Vol. 10, No. 11, 2001, pp. 1155-1163.

[11] L. Brenner, "Malathion," Journal of Pesticide Reform, Vol. 12, 1992, p. 29

[12] E. Bustos-Obregón and P. Gonzáles-Hormazabal, "Effect of a Single Dose of Malathion on Spermatogenesis in Mice," Asian Journal of Andrology, Vol. 5, No. 2, 2003, pp. 105-107.

[13] M. Abdollahi, M. Donyavi, S. Pournourmohammadi and M. Saadat, "Hyperglycemia Associated with Increased Hepatic Glycogen Phosphorylase and Phosphoenolpyruvate Carboxykinase Activities in Rats Following Subchronic Exposure to Malathion," Comparative Biochemistry and Physiology Part C: Pharmacology, Toxicology and Endocrinology, Vol. 137, No. 4, 2004, pp. 343-347.

[14] T. A. Slotkin, "Developmental Cholinotoxicants: Nicotine and Chlorpyrifos," Environmental Health Perspectives, Vol. 107, Suppl. 1, 1999, pp. 71-80.

[15] P. Z. Ruckart, K. Kakolewski, F. J. Bove and W. E. Kaye, "Long-Term Neurobehavioral Health Effects of Methyl Nparathion Exposure in Children in Mississippi and Ohio," Environmental Health Perspectives, Vol. 112, No. 1, 2004, pp. 46-51. doi:10.1289/ehp.6430

[16] Sanborn, Margaret, et al., "Systematic Review of Pesticide Human Health Effects," Ontario College of Family Physicians Toronto, Toronto, 23 April 2004.

[17] F. L. Assini, K. D. Zanette, P. S. Brocardo, P. Pandolfo, A. L. Rodrigues and R. N. Takahashi, "Behavioral Effects and ChE Measures after Acute and Repeated Administration of Malathion in Rats," Environmental Health Perspectives, Vol. 20, No. 3, 2005, pp. 443-449. doi:10.1016/j.etap.2005.05.007

[18] C. I. Acker, A. C. Souza, S. Pinton, J. T. Da Rocha, C. A. Friggi, R. Zanella and C. W. Nogueira, "Repeated Malathion Exposure Induces Behavioral Impairment and AChE Activity Inhibition in Brains of Rat Pups," Ecotoxicology and Environmental Safety, Vol. 74, No. 8, 2011, pp. 2310-2315. doi:10.1016/j.ecoenv.2011.07.035

[19] T. A. Slotkin, T. L. Lassiter, I. T. Ryde, N. Wrench, E. D. Levin and F. J. Seidler, "Consumption of a High-Fat Diet in Adulthood Ameliorates the Effects of Neonatal Para- thion Exposure on Acetylcholine Systems in Rat Brain Regions," Environmental Health Perspectives, Vol. 117, No. 6, 2009, pp. 916-922.

[20] S. X. Guo-Ross, , J. E. Chambers, , E. C. Meek and R. L. Carr, "Altered Muscarinic Acetylcholine Receptor Subtype Binding in Neonatal Rat Brain Following Exposure to Chlorpyrifos or Methyl Parathion," Toxicological Sciences, Vol. 100, No. 1, 2007, pp. 118-127. doi:10.1093/toxsci/kfm195

[21] J. Tang, R. L. Carr and J. E. Chambers, "The Effects of Repeated Oral Exposures to Methyl Parathion on Rat Brain Cholinesterase and Muscarinic Receptors during Postnatal Development," Toxicological Sciences, Vol. 76, No. 2, 2003, pp. 400-406. doi:10.1093/toxsci/kfg245

[22] F. O.Johnson, J. E. Chambers, C. A. Nail, S. Givaruangsawat and R. L. Carr, "Developmental Chlorpyrifos and Methyl Parathion Exposure Alters Radial Arm Maze Performance in Juvenile and Adult Rats," Toxicological Sciences, Vol. 109, No. 1, 2009, pp. 132-142. doi:10.1093/toxsci/kfp053

[23] L. M. Icenogle, N. C. Christopher, W. P. Blackwelder, D. P. Caldwell, D. Qiao, F. J. Seidler, T. A. Slotkin and E. D. Levin, "Behavioral Alterations in Adolescent and Adult Rats Caused by a Brief Subtoxic Exposure to Chlorpyrifos during Neurulation," Neurotoxicology and Teratology, Vol. 26, No. 1, 2004, pp. 95-101. doi:10.1016/j.ntt.2003.09.001

[24] A. Ennaceur, "One-Trial Object Recognition in Rats and Mice: Methodological and Theoretical Issues," Behavioural Brain Research, Vol. 215, No. 2, 2010, pp. 244 254.

[25] A. Ennaceur and J. Delacour, "A New One-Trial Test for Neurobiological Studies of Memory in Rats. 1: Behavioral Data," Behavioural Brain Research, Vol. 31, No. 1, 1988, pp. 47-59 doi:10.1016/0166-4328(88)90157-X

[26] C. A. Barnes, "Memory Deficits Associated with Senescence: A Neurophysiological and Behavioral Study in the Rat," Journal of Comparative \& Physiological Psychology, Vol. 93, No. 1, 1979, pp. 74-104.

[27] I. Fedorova, N. Hussein, M.H. Baumann, C. Di Martino and N. Salem, Jr. "An n-3 Fatty Acid Deficiency Impairs Rat Spatial Learning in the Barnes Maze," Behav Neurosci. Vol. 123, No. 1, 2009, pp. 196-205. doi: $10.1037 / \mathrm{a} 0013801$

[28] U. Greferath, A. Bennie, A. Kourakis and G. L. Barrett, "Impaired Spatial Learning in Aged Rats Is Associated with Loss of p75-Positive Neurons in the Basal Forebrain," Neuroscience, Vol. 100, No. 2, 2000, pp. 363-373. doi:10.1016/S0306-4522(00)00260-8

[29] J. M. Daniel, A. J. Fader, A. L. Spencer and G. P. Dohanich, "Estrogen Enhances Performance of Female Rats during Acquisition of a Radial Arm Maze," Hormones and Behavior, Vol. 32, No. 3, 1997, pp. 217-225.

[30] D. S. Olton, "The Radial Arm Maze as a Tool in Behavioral Pharmacology," Physiology \& Behavior, Vol. 40, No. 6, 1987, pp. 793-797. doi:10.1016/0031-9384(87)90286-1

[31] G. L. Ellman, K. D. Courtney, V. R. M. Andres and R. M. 
Featherstone, "A New and Rapid Colorimetric Determination of Acetylcholinesterase Activity," Biochemical Pharmacology, Vol. 7, No. 2, 1961, pp. 88-95. doi:10.1016/0006-2952(61)90145-9

[32] F.-Z. Azzaoui, H. Hami, M. El-Hioui, S. Boulbaroud and A. Ahami "Attempt at the Determination of Aluminum Nitrate LD50 and the Study of Its Neurotoxicological Effect in Wistar Rat," Biology and Medicine, Vol. 4, No. 2, 2012, pp. 89-94.

[33] H. R. Santos, W. M. Cintra, Y. Aracava, C. M. Maciel, N. G. Castro, E. X. Albuquerque, "Spine Density and Dendritic Branching Pattern of Hippocampal CA1 Pyramidal Neurons in Neonatal Rats Chronically Exposed to the Organophosphate Paraoxon," Neurotoxicology, Vol. 25, No. 3, 2004, pp. 481-494.

[34] A. L. Jones and L. Karalliedde, "Poisoning," In: N. A. Boon, N. R. Colledge, S. S. Davidson and B. R. Walker, Eds., Davidson's Principles and Practice of Medicine, 20th Edition, Churchill Livingstone, Edinburgh, 2006, pp. 203-226.

[35] M. Antunes and G. Biala. "The Novel Objects Recognition Memory: Neurobiology, Test Procedure, and Its Modifications," Cognitive Processing, Vol. 13, No. 2, 2012, pp. 93-110. doi:10.1007/s10339-011-0430-Z

[36] M. L. Reger, D. A. Hovda and C. C. Giza, "Ontogeny of Rat Recognition Memory Measured by the Novel Object Recognition Task," Developmental Psychobiology, Vol. 51, No. 8, 2009, pp. 672-678. doi:10.1002/dev.20402

[37] T. T. Win-Shwe, D. Nakajima, S. Ahmed and H. Fujimaki, "Impairment of Novel Object Recognition in Adulthood after Neonatal Exposure to Diazinon," Archives of Toxicology, Vol. 87, No. 4, 2012, pp. 753-762.

[38] R. S. Hammond, L. E. Tull and R. W. Stackman, "On the Delay-Dependent Involvement of the Hippocampus in Object Recognition Memory," Neurobiology of Learning and Memory, Vol. 82, No. 1, 2004, pp. 26-34. doi:10.1016/j.nlm.2004.03.005

[39] J. R. Clarke, M. Cammarota, A. Gruart, I. Izquierdo and J. M. Delgado-Garcia, "Plastic Modifications Induced by Object Recognition," Proceedings of the National Academy of Sciences of the United States of America, Vol. 107, No. 6, 2010, pp. 2652-2657. doi:10.1073/pnas.0915059107

[40] B. D. Winters and T. J. Bussey, "Removal of Cholinergic Input to Perirhinal Cortex Disrupts Object Recognition but Not Spatial Working Memory in the Rat," European Journal of Neuroscience, Vol. 21, No. 8, 2005, pp. 22632270. doi:10.1111/j.1460-9568.2005.04055.x

[41] C. F. Hohmann, "A Morphogenetic Role for Acetylcholine in Mouse Cerebral Neocortex," Neuroscience \& Biobehavioral Reviews, Vol. 27, No. 4, 2003, pp. 351-363. doi:10.1016/S0149-7634(03)00066-6

[42] J. Yanai, "Neurobehavioral Teratology," Elsevier, Amsterdam, 1984.

[43] T. A. Slotkin, "Cholinergic Systems in Brain Development and Disruption by Neurotoxicants: Nicotine, Environmental Tobacco Smoke, Organophosphates," Toxicology and Applied Pharmacology, Vol. 198, No. 2, 2004, pp. 132-151. doi:10.1016/j.taap.2003.06.001

[44] B. Eskenazi, A. Bradman and R. Castorina, "Exposures of Children to Organophosphate Pesticides and Their Potential Adverse Health Effects," Environmental Health Perspectives, Vol. 107, Suppl. 3, 1999, pp. 409-419. doi:10.1289/ehp.99107s3409

[45] G. Sarkisyan and P. B. Hedlund, "The 5-HT7 Receptor Is Involved in Allocentric Spatial Memory Information Processing," Behavioural Brain Research, Vol. 202, No. 1, 2009, pp. 26-31. doi:10.1016/j.bbr.2009.03.011

[46] T. A. Slotkin and F. J. Seidler, "Developmental Exposure to Terbutaline and Chlorpyrifos, Separately or Sequentially, Elicits Presynaptic Serotonergic Hyperactivity in Juvenile and Adolescent Rats," Brain Research Bulletin, Vol. 73, No. 4-6, 2007, pp. 301-309.

doi:10.1016/j.brainresbull.2007.04.004

[47] J. E. Aldridge, E. D. Levin, F. J. Seidler and T. A. Slotkin, "Developmental Exposure of Rats to Chlorpyrifos Leads to Behavioral Alterations in Adulthood, involving Serotonergic Mechanisms and Resembling Animal Models of Depression," Environmental Health Perspectives, Vol. 113, No. 5, 2005, pp. 527-531.

[48] G. Koopmans, A. Blokland, P. van Nieuwenhuijzen and J. Prickaerts, "Assessment of Spatial Learning Abilities of Mice in a New Circular Maze," Physiology \& Behavior, Vol. 79, No. 4-5, 2003, pp. 683-693. doi:10.1016/S0031-9384(03)00171-9

[49] T. Nakashiba, D. L. Buhl, T. J. McHugh and S. Tonegawa, "Hippocampal CA3 Output is Crucial for Ripple-Associated Reactivation and Consolidation of Memory," Neuron, Vol. 62, No. 6, 2009, pp. 781-787.

[50] H. Eichenbaum, "Cortico-Hippocampal System for Declarative Memory," Nature Reviews Neuroscience, Vol. 1, No. 1, 2000, pp. 41-50.

[51] S. Brimijoin and C. Koenigsberger, "Cholinesterases in Neural Development: New Findings and Toxicologic Implications," Environmental Health Perspectives, Vol. 107, Suppl. 1, 1999, pp. 59-64.

[52] J. W. Bigbee, K. V. Sharma, J. J. Gupta and J. L. Dupree, "Morphogenic Role for Acetylcholinesterase in Axonal Outgrowth during Neural Development," Environmental Health Perspectives, Vol. 107, Suppl. 1, 1999, pp. 81-87.

[53] M. Grifman, N. Galyam, S. Seidman and H. Soreq, "Functional Redundancy of Acetylcholinesterase and Neuroligin in Mammalian Neuritogenesis," Proceedings of the National Academy of Sciences of the United States of America, Vol. 95, No. 23, 1998, pp. 13935-13940. doi:10.1073/pnas.95.23.13935

[54] D. Qiao, F. J. Seidler, Y. Abreu-Villaça, C. A. Tate, M. M. Cousins and T. A. Slotkin, "Chlorpyrifos Exposure during Neurulation: Cholinergic Synaptic Dysfunction and Cellular Alterations in Brain Regions at Adolescence and Adulthood," Brain Research. Developmental Brain Research, Vol. 148, No. 1, 2004, pp. 43-52.

[55] A. D. Campaña, F. Sanchez, C. Gamboa, M, J. de Gómez-Villalobos, F. De La Cruz, S. Zamudio and G. Flores, "Dendritic Morphology on Neurons from Prefrontal Cortex, Hippocampus, and Nucleus Accumbens Is 
Altered in Adult Male Mice Exposed to Repeated Low Dose of Malathion," Synapse, Vol. 64, No. 4, 2008, pp. 283-290. doi:10.1002/syn.20494

[56] G. Sarkisyan and P. B. Hedlund, "The 5-HT7 Receptor Is Involved in Allocentric Spatial Memory Information Processing," Behavioural Brain Research, Vol. 202, No. 1, 2009, pp. 26-31. doi:10.1016/j.bbr.2009.03.011

[57] E. D. Levin, N. Addy, A. Nakajima, N. C. Christopher, F. J. Seidler and T. A. Slotkin, "Persistent Behavioral Consequences of Neonatal Chlorpyrifos Exposure in Rats," Developmental Brain Research, Vol. 130, No. 1, 2001, pp. 83-89. doi:10.1016/S0165-3806(01)00215-2

[58] R. P. Kesner, "Subregional Analysis of Mnemonic Functions of the Prefrontal Cortex in the Rat," Psychobiology, Vol. 28, No. 2, 2000, pp. 219-228.

[59] A. Louilot, K. Taghzouti, H. Simon and M. Le Moal, "Limbic System, Basal Ganglia, and Dopaminergic Neurons. Executive and Regulatory Neurons and Their Role in the Organization of Behavior," Brain, Behavior and Evolution, Vol. 33, No. 2-3, 1989, pp. 157-161. doi:10.1159/000115920

[60] E. J. Nestler and W. A. Carlezon Jr., "The Mesolimbic Dopamine Reward Circuit in Depression," Biological Psychiatry, Vol. 59, No. 12, 2006, pp. 1151-1159. doi:10.1016/i.biopsych.2005.09.018

[61] S. B. Floresco, S. Ghods-Sharifi, C. Vexelman and O. Magyar, "Dissociable Roles for the Nucleus Accumbens Core and Shell in Regulating Set Shifting," The Journal of Neuroscience, Vol. 26, No. 9, 2006, pp. 2449-2457.

\section{doi:10.1523/JNEUROSCI.4431-05.2006}

[62] T. A. Slotkin, B. E. Bodwell, E. D. Levin and F. J. Seidler, "Neonatal Exposure to Low Doses of Diazinon: Long-Term Effects on Neural Cell Development and Acetylcholine Systems," Environmental Health Perspectives, Vol. 116, No. 3, 2008, pp. 340-348. doi:10.1289/ehp. 11005

[63] S. Ghods-Sharifi, D. M. Haluk and S. B. Floresco, "Differential Effects of Inactivation of the Orbitofrontal Cortex on Strategy Set-Shifting and Reversal Learning," Neurobiology of Learning and Memory, Vol. 89, No. 4, 2008, pp. 567-573. doi:10.1016/j.nlm.2007.10.007

[64] R. N. Cardinal, J. A. Parkinson, J. Hall and B. J. Everitt, "Emotion and Motivation: The Role of the Amygdala, Ventral Striatum, and Prefrontal Cortex," Neuroscience \& Biobehavioral Reviews, Vol. 26, No. 3, 2002, pp. 321-352. doi:10.1016/S0149-7634(02)00007-6

[65] I. Toni and R. E. Passingham, "Prefrontal-Basal Ganglia Pathways Are Involved in the Learning of Arbitrary Visuomotor Associations: A PET Study," Experimental Brain Research, Vol. 127, No. 1, 1999, pp. 19-32. doi:10.1007/s002210050770

[66] M. G. Purisai, A. L. McCormack, S. Cumine, J. Li, M. Z. Isla and D. A. Di Monte, "Microglial Activation as a Priming Event Leading to Paraquat-Induced Dopaminergic Cell Degeneration," Neurobiology of Disease, Vol. 25, No. 2, 2007, pp. 392-400. doi:10.1016/j.nbd.2006.10.008 